tice; one which, even after all, is, I find, not quite novel, as, in the last century, Dr. Percival tried in phthisis pulmonalis the inspiration of fixed air or carbonic acid "by inhaling the steams of an effervescing mixture of chalk and vinegar, or of vinegar and potash", and Drs. Lettsom, Withering, and Hulme tried a similar method.

9. External Wounds and Burns. Seeing, first, the great and speedy relief to pain in cancerous and other sores obtained through the local application of carbonic acid by Ewart and Ingen-Housz; and, second, the tendency to cicatrisation observed even in some malignant ulcers when carbonic acid was kept in contact with them, I ventured in my paper on the subject to suggest the topical employment of carbonic acid to surgical wounds and burns, as at once both relieving suffering, and being one of the best means for producing rapid healing and cicatrisation. If found successful, it would not be difficult to devise simple means of applying it as a constant dressing. But no sufficient experiments, so far as I am aware, have been as yet made upon the matter.

10. Extraction of Teeth. I would suggest that perhaps carbonic acid may possibly be yet found useful as a local anæsthetic under a different form, and in a different mode from those already alluded to. Teeth have been now often extracted without pain when the gum was frozen with ice and salt, or ice and muriate of ammonia. But the application of these freezing mixtures, is tedious, difficult, and uncertain. If the chemist could show us a safe, simple, and cheap means of making and keeping solid carbonic acid, would not the application of the solid flakes of carbonic acid freeze the gum (or, indeed, any other part to which it was applied) sufficiently to produce the necessary degree of local anæsthesia?

In the preceding remarks I have scarcely referred, except incidentally, to the question of the utility of carbonic acid as a local anæsthetic when applied to various mucous surfaces and exposed external structures. Let me only in the meantime add, that all my subsequent experience has, in my opinion, more than confirmed the views which I ventured to publish two or three years ago with regard to its practical utility and efficiency as a local sedative or anodyne agent. The late observations of Drs. Churchill, Johns, etc., in our own country, and of Follin, Broca, Bernard, etc., in France, all tend further to prove and establish the advantages to be often obtained in practice from the employment of this therapeutic agent as a topical anæsthetic.

\section{ON SOME SEVERE FORMS OF DISEASE ARISING FROM THE RETENTION OF DECAYED TEETH.}

By J. C. Clendon, Esq., Surgeon, Lecturer on Dental Surgery at the Westminster Hospital.

[Iicad before the Greenwich Medical Society.]

[Coneluded from page 449.]

I MIGHT mention more evils resulting from the retention of diseased teeth, but they would unduly lengthen this paper; I will, therefore, at once proceed to read a few cases I have selected from my notes, to illustrate those I have already pointed out.

CaSe I. Disease in Antrum. Mr. D., a gentleman about thirty-five years of age, an architect, of tolerable health, but who suffered occasionally from chronic catarrh, had during the last six months expectorated a considerable quantity of fotid pus or mucus mixed with blood on rising from his bed in the morning. Having lost several members of his family from tubercular disease, he naturally became uneasy, and consulted his usual medical attendant, who prescribed for his cough, but failed to discover the source of the complaint.

He subsequently saw Mr. Holt, one of the surgeons to the Westminster Hospital, who, from the history of the case, at once suspected the cause of the malady, and recommended him to apply to me that his teeth might be thoroughly and carefully examined. The teeth seemed in tolerable order, and quite free from pain; but there was one, the first superior molar, that attracted my notice, not so much from the fact of its having been stopped, as from its displacement from the line of the neighbouring teeth, and its tenderness on pressure. $\mathrm{He}$ allowed me to extract it, when I found a fibrous growth of the size of a hazel-nut attached by a pedicle to the extremity of the root. This growth had made room for itself by penetrating the floor of the antrum, and by its presence there had set up inflammation in the lining membrane. The sanguineo-puru- lent secretion, the result of the inflammation, accumulating and filling the antrum during the day, found its way during sleep, when the body was in a recumbent posture, through the natural opening into the middle meatus of the nose, and thence by the posterior nares into the throat, to be coughed up on the patient's resuming the erect position in the morning.

No pus followed the removal of the tooth, proving that the tumour was under the membrane, and not actually within the antrum. I pierced the membrane with a trocar, and found it thick, tough, and difficult to penetrate; but when it was freely open, a tablespoonful or more of very fœetid pus made its escape.

I washed out the antrum with warm water, and injected it occasionally with a weak solution of sulphate of zinc. In the course of three weeks or a month, to the great relief of the patient's mind, the discharge (and the cough) had entirely subsided.

CASE Ir. Tumour: an Encysted Abscess in the Cheek. Mary Overall, aged 21, waistcoat-maker, a healthy, good looking young woman, with a tumour or swelling of the right cheek, came under my care, last November, at the Westminster Hos. pital. The application was well-timed, for the tumour fluctuated on pressure, and seemed, from its nipple-like protrusion, just about to burst outwardly; the skin over it was tense, but not at all discoloured. On grasping the tumour with a finger and thumb placed inside and outside the cheek, a thickened sac, of about the size and form of an oyster, could easily be traced in a position corresponding with the buccinator muscle. In the upper jaw were the roots of two decayed teeth, a second bicuspid and a first molar, but the patient was " quite sure these had nothing to do with it, as she had never had pain in them, and the swelling had been coming on gradually during the last twelve months from a cold caught in the face, while crossing over from Dublin." I thought it better to remove these teeth notwithstanding, when through the opening thus made the sac immediately emptied itself and filled the mouth. The pressure of the tumour in the cheek or of the pus in the an trum, or both together, had led to the destruction of the outer wall of the maxilla; the gum hung loosely over the cavity, and I could easily pass my finger under it into the antrum. The sac was exceedingly thick; it has taken months to absorb, but is now disappearing, and the cavity in the jaw is filling up while, the face being naturally round and plump, I do not anticipate any permanent deformity.

CASE III. Facial Neuralgia, or true Tic Douloureux. On my arrival in town one morning I was hastily summoned to a lady suffering from facial neuralgia. She was about thirty-four years of age, tall and handsome, the wife of an official in one of the Indian presidencies. She had a young family, and, with the exception of severe periodical attacks of tic douloureux, her health was good. The attacks in question were attributed to ague, intermittent fever, and long residence in India ; but after having heard her own history of the case, and having had an opportunity of examining her mouth, I referred them to a totally different cause.

On the occasion in question the attack had come on suddenly during the night, and at the time of my visit the eye was quite closed, and the face and head so swollen, that it was impossible to recognise her; she looked as if she had been suffering some days from erysipelas. She was attended by an old friend, Dr. Wallace, of Carshalton, a retired medical officer of the Indian army. Expressing my conviction that two molar teeth, which I pointed out, were the sole cause of her suffering, Dr. Wallace concurred with me in the propriety of my removing them; a proceeding to which the patient for some time objected, as "the teeth had been stopped for several years, and had not given her pain or uneasiness." They were, however, removed; the pain immediately ceased, and by the next day all the symptoms had disappeared.

In this case, the pain arose entirely from the deposit of cementum in a cavity not large enough to contain it. I preserved and made sections of the teeth in question, and they are here for your inspection. You will perceive they have both been stopped with ordinary amalgam, but that the arrest of the decay is due to the cementum; it surrounds and blocks th decay, entirely fills up the cavity that originally contained the pulp, and envelopes in a solid mass the three roots of the teeth, the ivory-like structure of the latter being easily traced in the midst of the surrounding bone. Now, there was not a trace of vascular tissue in or about the tooth, save in the periosteum enveloping the bone; and to the pressure of the deposit on the periosteum, and the resistance offered to it by the surrounding alveolus, I attribute all the suffering and its results. 
At all events, the removal of the teeth effected a cure. She had no return of the pain while in England; and some time after her arrival in India she wrote, as she said, "gratefully to tell me she had had no return of the malady."

CASE IV. Neuralgia, from Deposit of Bone in the Jaw. J.A., about fifty years of age, a house carpenter, had suffered so constantly from facial neuralgia for two years, that he had been unable to follow his usual occupation; he had been under private medical treatment, and also at a dispensary in Pimlico, and was then admitted at the Westminster Hospital under the care of Dr. Kingston. Now, Dr. Kingston is one who believes in the local origin of the great majority of these complaints, and makes a point never to order medicine until the mouth has been carefully and thoroughly examined by some one in whom he has confidence; and with this view he brought the patient to me.

I found the teeth were, as the patient averred, all sound and perfect in their arrangement, but there was one, a molar tooth, more sensitive on percussion than its fellows, and that tooth I extracted. The cause of the evil was manifest; the roots were being gradually absorbed, owing to pressure in the socket; they presented just the same appearance as the roots of the temporary when removed by the advance of the permanent teeth. No medicine was needed or giver. He called on me at the Hospital some months afterwards, to say he had had no repetition of the pain; and as I have not had a visit from him since, I presume he continues well.

CASE v. Neuralgia, with Permanent Contraction of some of the Facial Muscles. A young lady, the daughter of a clergyman, at school at Kensington, was brought to me by request of her family medical attendant, Mr. Robert Ceely, of Aylesbury. She was about sixteen years of age, and very tall, having grown rapidly. For the last two years she had suffered incessantly with her teeth, and on the right side of the face some of the muscles were affected by the constant irritation, the corner of the mouth being drawn up, and the eyelids contracted. I found her mouth full of decayed and decaying teeth; with the exception of the six lower front and the two canines in the upper jaw, they were all so much diseased as not, under any circumstances, to be worth preserving; but seeing how long she had suffered, and was likely to do as long as the vestige of a diseased tooth remained, I recommended their instant removal. This was a sad alternative for a young lady of sixteen, but the necessity for the step was self-evident; for her own part, "she had suffered so much that she was only too glad to get rid of them " and there is no doubt that, apart from other considerations, healthy gums are far more useful for the purposes of mastication than tender and decayed teeth. All the decayed teeth were removed under chloroform in two operations; and when the gums were sufficiently healed artificial teeth were substituted. Three years have now elapsed since the operations; during the interval I have seen her occasionally; she has remained quite free from pain; but some amount of the old deformity, arising from the contraction of the muscles, still remains, and is, I fear, permanent.

CASE vr. Abscesses in the Face. A respectable tradesman in the country came to town for advice, and was recommended by his medical attendant to call on me. The patient, a steady man, of spare habit, about forty years of age, had been troubled during the previous twelve months with a succession of abscesses in the left side of the face. These, as they formed, had been fomented and poulticed, and in due time had either been opened by lancing, or made an opening for themselves through the integuments of the face. At the time of his first visit there were four ragged orifices through which small quantities of pus were continually oozing, and into either of which the little finger would enter, while the surrounding in tegument was thickened and boggy to the touch, with that peculiar discoloration which is so characteristic of the presence of diseased bone. Although the orifices were widely separated, a probe passed into either led to one spot only, namely, to the posterior molar teeth of the upper jaw; and in that locality I found two or three diseased roots deeply imbedded in a thickened mass of fungoid gum. These roots I removed, together with several small portions of carious bone.

When I last saw him, six weeks or two months after the operation, two orifices were still open and discharging pus, and small portions of carious bone were occasionally coming away. The closed orifices have, in healing, contracted, and bound the integument to the subjacent tissue, each one forming a cup like depression with a ragged cicatrix in the centre, the cheek presenting the appearance of having been riddled by musket balls.
In this case, had I been aware of the extent cf the diseased bone, I should, in the first instance, have urged its entire removal.

CASE vir. Abscess and Paralysis of the Face. R. W., aged 34 , harness-maker, gave me the following history :- "In January last (1857) I felt pain in the ear, which began to discharge; then an abseess formed in front of the ear; this was lancell at the Northern Dispensary, and it continued to discharge freely for a week or more. Having lost the hearing on that side, $I$ went to the Ear Infirmary in Soho Square for two months, without any benefit. Discharge of the abscess through the ear continued until December. I then first began to feel severe pain when I attempted to move the jaw; this was followed by inability to close the eye, or to put out my tongue. In that state I came to this (the Westminster) Hospital in January last, and was admitted under the care of Dr. Reynolds."

From the constant pain and tenderness on pressure, Dr. Reynolds, suspecting some local cause of irritation, sent him to me, in order that his mouth might be carefully examined. When I first saw him, there was great difficulty in opening his mouth; severe pain in the condyle when he attempted to move the lower jaw; on pressure being made in that direction; he was unable to close the eyelids, to contract the orbicular muscle of the eye, to inflate the cheek, or to protrude the tongue beyond the front teeth, gasping when he endeavoured to do it. On attempting to whistle, the mouth and nostrils were drawn to the opposite side; the external meatus of the ear was so contracted that a fine probe only would pass; and through the orifice, on pressure being made on the cheek, pus oozed out He had no toothache, nor decayed tooth that he knew of; but I found the teeth on the affected side encrusted with tartar, from want of use. This to me is always a suspicious circumstance. He attributed it to pain in the hinge of the jaw in eating, and to his inability to remove the food from the cheek, owing to the paralysis of the muscles. On passing a curved instrument under the gum between two molar teeth, he felt some pain in one tooth; and, repeating the experiment with the same result, I determined to remove it. There was a cavity in it, quite out of sight, and almost out of reach, through which, I have no doubt, air had from the first freely passed, and given rise to all the mischief. On washing out the mouth, he at once expressed a sense of relief; found he could open his mouth easily; and, to the surprise of all present, he could protrude his tongue to the full extent without any apparen effort, and also partially close the eye. The relief might be described as instantaneous. The inability to contract the muscles of the mouth, to inflate both cheeks, and raise the angle of the mouth, still continues; and, although it is asserted that in the severest lesions the nerves do not slough, I nevertheless expect some branches of the portio dura have been destroyed in the long continued abscess, and that loss of power in some of the muscles, as well as of hearing, on that side, are permanent and irremediable.

CASE vir. Disease of Bone and Abscess in the Face, from Retention of Diseased Teeth. The last case I will bring under your notice is one from this immediate neighbourhood, and it has attracted some little attention. The patient was Thomas Harcourt, aged 45, carpenter, of St. Jokn's Terrace, Lewisham Road. I will give the history in his own words.

"In January 1857, I went to bed quite well, and, when I awoke in the morning, I found my jaw was closed and my teeth clenched. I could not open my mouth. I had no tooth ache, nor bad teeth that I knew of, but fancied it was owing to the dampness of the house. I called on Dr. Edward Downing, who gave me some medicine. My face began to swell, and a large abscess to form in the cheek in front of the ear. I was told to poultice it. In about a month or so, it burst of itself, and the matter escaped out of my mouth between the front teeth. The discharge continued night and day for about three months; I should think, as much as a teacupful in twentyfour hours. In April I went to Guy's Hospital, and remained an out-patient for about a month, under the care of one of the assistant-surgeons, who gave me an ointment for my face, and some medicine to swallow; but, getting no better, I left off going. During the whole of this time, my mouth had never been opened. I lived upon suction, my teeth being clenched. I had done no work since January last; I was too ill to do any. I saw Dr. Edward Downing again, and, about the end of July, he gave me his card, and desired me to call on you at the Westminster Hospital."

This was his history. At the time of his calling on me, his countenance was sallow, and he was much emaciated: not at all 
surprising, when the fact of his not having tasted solid food for six months, and the amount of the discharge during the same period, are taken into consideration. He was equally depressed in spirits. He had been prayed for in church for some months, and seemed to have made up his mind he could not live much longer. The first thing to do was, what had been so long neglected, namely, to open his mouth to make an examination; and this I effected with a series of screws and wedges, gradually increasing in size, and applied at the same moment on both sides of the mouth. I had offered him chlo. roform, but he declined, saying "he was ready to bear any amount of pain likely to do him good". It must have been severe, but he did bear it, manfully. On passing in my finger, the cause of the mischief was at once evident: two superior molar teeth, decayed and dead, lay imbedded in a portion of necrosed bone; the whole firmly held in situ by the closure of the jaw, and no effort of nature could ever have removed it, save through the abscess in the cheek. An elevator separated the mass, and turned it out of the mouth without much difficulty. After washing out his mouth, he found he could voluntarily open it to the same extent I had forced it. This is a remarkable feature in these cases. I used to imagine the rigidity was due to the ligaments of the joint, but I am now quite satisfied it is owing to the contraction of the masseter or internal pterygoid muscle, the weak depressors of the lower jaw being quite useless to antagonise it; and the moment the cause of irritation is removed, the muscle is relaxed, and the jaw opens freely.

About three weeks subsequently, a piece more bone exfoli ated; and from that time his recovery was rapid. I saw him last week. His health seems good. There is a slight depression in the cheek, corresponding to the loss of the bone, but nothing approaching to a deformity.

Now, in all these cases, whatever the symptoms, and whether the pain be severe, mitigated, or altogether absent, there is throughout but one indication-namely, an effort of nature to get rid of the offending body, which we, if we would endeavour wisely to assist and second instead of to counteract her, ought at once to seek out and remove.

But here is the difficulty. The patient has a dread of the operation, and will run all ris ks, submit to any other course of treatment, or any amount of tsuffering, rather than undergo it. An old lady of seventy once old me that, from her earliest recollection down to the period when the last remaining tooth had worked its own way out, she had seldom been free from pain for a month together; and yet she could never summon courage to submit to the operation of extraction: in her own emphatic words, "she felt she must die first". But in severe cases, where there is great tenderness or pain, or where the operation would be more than usually painful and difficult to perform, chloroform will deprive the patient of all excuse, and prove a great blessing. It allows the practitioner to open and examine the mouth carefully, perhaps for the first time; and then, at his leisure, to seek for and remove all that he deems necessary. It is true that, owing to some few casualties, which from the first I anticipated, some persons have as much dread of chloroform as of the operation itself; but my experience in some three thousand cases, taken indiscriminately, and extending over a period of more than ten years, satisfies me that although, like all powerful agents, it is dangerous if misused, it can always be given with safety if administered with proper care. I can truly aver that, until the other day, when, in a case of protracted operation at the shoulder-joint, much blood being lost, syncope came on while the patient was fully under the influence of the chloroform, but which was detected and counteracted on the instant, I have not had one case which, during its administration or subsequently, ever gave me a moment's uneasiness.

Often, however, there is another difficulty: and that is on the part of the practitioner. Perhaps he examines the mouth, and ascertains the cause of the pain to be the root of a tooth broken off and buried deeply in the gum, or he finds a number of roots clustered together, presenting no tangible surface for the grasp of an instrument, and difficult to reach even with an elevator. He sees they ought to be removed, but shrinks from the task. To use a familiar phrase, "he does not like the look of them"; and therefore recommends some palliative mode of treatment, and patience. In point of fact, from not feeling quite equal to the emergency, or wanting confidence in the use of the instrument, he is reluctantly compelled to leave his patient to an indefinite period of suffering, and perhaps to some of the evil results $I$ have already pointed out. This abnegation of his legitimate functions, arising purely from want of confidence in himself, has notoriously had the effect of driving this department of surgery into the hands of unqualified and often uneducated practitioners, styling themselves surgeondentists, and affecting to consider the mouth as their own legitimate domain, to which the surgeon has no claim. But, whatever the cause, the result is the same; for now the old story is repeated; the cheek swells, pus forms, and there is either a circumscribed or diffused abscess. This is poulticed, to promote absorption, or to assist resolution, as the case may be. Now, I cannot too forcibly insist that fomenting the cheek under such circumstances is the most erroneous step of all for, should the abscess point outwardly, there will be a sinus from which pus may continue to flow, perhaps for a year or more, as long as there is any root or carious bone to exfoliate, leaving, when healed, a deep pit or cicatrix in the cheek, which disfigures the patient for life. This is not a pleasant alternative for a man, but a very serious drawback to a woman, in the preservation of whose good looks we all feel a natural interest. The tooth should be removed, when the pus will immediately flow through the aperture thus made; or, failing that, hot water, or hot bread and water fomentations, should be used in the mouth; and, as soon as possible, the abscess, however deeply seated, should be opened freely through the mucous membrane, and the pus allowed to escape into the mouth.

In conclusion, I think no one will deny that it should be a part of a medical man's education to know how to deal with such cases; and I trust that all you who have kindly gone with me through these observations will have felt their interest and importance; and, seeing how much suffering and mischief may spring from so small a cause as a diseased tooth, you will sympathise in my earnest wish to obtain the diffusion of more enlightened views on this much neglected, and therefore little understood department of surgery. Every day confirms my experience of its necessity, and strengthens my desire to see it accomplished. Many complaints, that come under the notice of the practitioner as diseases of the body, are in reality diseases of the teeth. It is clearly impossible to treat the body as a whole, if we are ignorant of its parts; and I will venture to say there is no part much more widely and universally affecting the general system than that of the teeth.

\section{APPLICATION OF BELLADONNA AS AN ANTILACTESCENT.}

By Henry G. Trend, Esq.

In the JOURNAL of May 29th last, some very interesting re marks appeared upon the use of belladonna in checking the secretion of milk, from the pen of Mr. Newman of Fulbeck, near Grantham; and as $I$ am in a position to clear up some few doubts which that gentleman seems to entertain as to it effect upon one breast only, and also upon the infant, I have thought it worth while to make a few observations thereon

In the early part of this year, I had two cases in which I deemed it advisable to check the secretion of milk in one breast; one from threatening abscess, the other from excessively tender nipples. In both cases, I allowed the infant to continue at the other breast; there was an abundant supply of milk, and neither of the children had a single bad symptom. The breast operated upon, in each case, soon resumed its natural size.

I have used the belladonna now more than a dozen times, and have seen it fail but once; and, even in that case, it considerably relieved that intense suffering which lacteal abscess invariably causes. I firmly believe that if ladies would but mention the first precursory symptoms of these abscesses to their medical attendants, instead of to some meddlesome, ignorant old woman, "bad breasts" would, by the use of belladonna gradually be numbered among the things that were.

The Health of Paris. The Gazette Hebdomadaire de Médecine of June 4 th, says:-Paris presents at this time a striking example of the rapidity with which atmospheric changes exert their influence on the public health. The hot weather has scarcely set in more than five or six days; and already there have been met with, in private practice, a large proportion of cases of intestinal disorder, especially diarrhœea. We have taken care to ascertain that many of the persons attacked had not yet tasted any of the fruits of the season; there has therefore been a true constitutio medica, connected, there can be no doubt, with the elevation of the temperature. 